\title{
ORIGINAL
}

\section{EVALUACIÓN DEL PROCESO DE UN PROGRAMA REALIZADO A TRAVÉS DE INTERNET Y DE LA TELEFONÍA MÓVIL PARA PROMOVER CONDUCTAS SALUDABLES EN ESTUDIANTES DE EDUCACIÓN SECUNDARIA DE ESPAÑA Y MÉXICO (*)}

\author{
Alberto Lana Pérez (1), María José García Fernández (2) y María Luisa López González (1).
}

(1) Área de Medicina Preventiva y Salud Pública. Instituto Universitario de Oncología del Principado de Asturias. Facultad de Medicina y Ciencias de la Salud. Universidad de Oviedo.

(2) Programas internacionales. Dirección Prepa Tec. Instituto Tecnológico y de Estudios Superiores de Monterrey.

(*) Esta investigación fue financiada por el Instituto de Salud Carlos III (FISSPI080544) y ha contado con la inestimable colaboración institucional de la Universidad de Oviedo (España) y de la Subsecretaría de Educación Pública (Estados Unidos Mexicanos).

Los autores declaran no tener ningún conflicto de intereses

\section{RESUMEN}

Fundamentos: Internet y la telefonía móvil forman parte de la tecnología más reciente que puede utilizarse en la educación para la salud. E objetivo fue evaluar el proceso de un programa para prevenir conductas de riesgo de cáncer implementado a través de internet y telefonía móvil.

Métodos: Durante tres cursos académicos del período 2009-12 se seleccionó a estudiantes de secundaria de España y México para participar en un programa en línea suplementado con el envío de mensajes al teléfono móvil (SMS) cuyo objetivo era prevenir conductas de riesgo de cáncer. La intervención fue diseñada como un ensayo aleatorizado, con un grupo experimental (GE) y un grupo control. Se midieron el reclutamiento y la retención utilizando las herramientas de la plataforma de gestión de la web y de Google Analytics

Resultados: 3.855 estudiantes iniciaron el registro en el programa de los cuales $2.001(51,9 \%)$ completaron el cuestionario. 77,5\% fueron mexicanos, de 13 años $(40,6 \%)$, de buen nivel académico $(68,7 \%)$ y con padres $(49,6 \%)$ y madres $(53,9 \%)$ universitarios. El 56,4\% registró un número de teléfono para recibir SMS. El GE estuvo formado por 1.014 estudiantes y la media de sus visitas a la web fue 31,6 en el primer curso, 21,8 en el segundo y 21,9 en el tercero. Las duraciones medias fueron, respectivamente, 11:02, 8:07 y 12:55 minutos. Cada adolescente del GE logró incorporar a 1,16 adultos (total1.172) y otros 1.076 se registraron espontáneamente. La tasa de retención de los estudiantes del GE fue $41,5 \%$ al final del seguimiento y fue mayor entre quienes tenían mejor nivel académico (OR:12,5), eran mexicanos (OR:4,4), tenían 12 años (OR: 3,1$)$ y habían sido incorporados durante el primer trimestre del curso (OR: 2,8 ).

Conclusión: El reclutamiento y la retención de estudiantes fueron escasos, especialmente en España. No obstante, los estudiantes que participaron visitaron la web del programa, en teoría durante un tiempo suficiente para lograr buenos resultados preventivos.

Palabras clave: Adolescentes. Estudiantes. Internet. Salud escolar. Teléfono móvil. Educación para la Salud. Conducta de salud. Control de conducta. Neoplasias.

\section{Correspondencia}

Alberto Lana Pérez.

Facultad de Medicina y Ciencias de la Salud.

Avda. Julián Clavería s/n.

33006. Oviedo

España.

lanaalberto@uniovi.es

\section{ABSTRACT \\ Evaluation of Process of an Educational \\ Web-Based and Mobile Phone-Based Program for Encouraging Healthy Behaviours among Spanish and Mexican Students}

Background: Current communication technologies can be used in health education. The aim was to assess the process of an online program designed to prevent cancer risk behaviours using an educational website and mobile phones.

Methods: High school students from Spain and Mexico were recruited during 3 academic years (2009-12) to participate in a web-based program supplemented with mobile phone messages (SMS) which aim was to prevent cancer risk behaviours. The program was designed as a randomized trial, with control and experimental group (EG). Recruitment and adherence were analyzed using data of the Web management platform and Google Analytics.

Results: 3,855 students started the logging on the program of which $2,001(51.9 \%)$ completed the questionnaire. $77.5 \%$ were Mexicans, 13 years old $(40.6 \%)$, with good academic level $(68.7 \%)$ and with parents $(49.6 \%)$ and mothers $(53.9 \%)$ having university degree. $56.4 \%$ recorded a phone number to receive SMS. The EG consisted of 1,014 students and the averages of their visits to the website were 31.6 in the first year, 21.8 in the second and 21.9 in the third. Each adolescent of the EG was able to incorporate 1.16 adults (total 1,172) and other 1,076 were recorded spontaneously. Retention rate at the end of follow-up was $41.5 \%$ and was higher among those who were best students (OR: 12,5), Mexicans (OR: 4.4), 12 years old (OR: 3.1 ) and have been incorporated in the first three months of the implementation (OR: 2.8).

Conclusion: Students' recruitment and retention was scarce, mainly in Spain. However students involved visited the program website with sufficient amount of time to achieve good results.

Keyword: Adolescent. Health school. Students. Internet. Cellular Phone. Health Education. Health behaviour. Behaviour control. Neoplasms. 


\section{INTRODUCCIÓN}

España lleva años experimentando un leve pero sostenido descenso en las tasas de incidencia de cáncer y de su mortalidad ${ }^{1,2}$. En México la situación es incluso mejor, con tasas notablemente inferiores a las mundiales ${ }^{3}$. No obstante, en los países económicamente más desarrollados todavía se espera que alrededor de 1 de cada 2 hombres y 1 de cada 3 mujeres desarrollen al menos un tumor maligno ${ }^{4}$. Las conductas de riesgo para las principales enfermedades crónicas en adultos, como el cáncer, frecuentemente se inician y establecen durante la adolescencia. Estas conductas incluyen el consumo de sustancias como tabaco y alcohol, una autonomía creciente en la alimentación (con predominio de alimentos poco saludables) o el abandono de las actividades deportivas de la infancia 5 .

En la Unión Europea, la Asociación de Ligas Europeas Contra el Cáncer proporciona un marco común para la lucha contra esta enfermedad. Entre otras acciones, es responsable del Código Europeo contra el Cáncer (CECC), cuya cuarta revisión está en fase de diseño y que incluye siete conductas de prevención primaria del cáncer ${ }^{6}$. Como se basan en la misma evidencia científica, las recomendaciones de otros organismos suelen ser superponibles, como las de la Subsecretaría de Prevención y Promoción de la Salud de México ${ }^{7}$. La difusión de estos consejos es requerida por los gobiernos de España y México ${ }^{7,8}$ y puede realizarse entre la población adolescente y adulta mediante técnicas muy diversas. Entre estas, las intervenciones que tratan de modificar simultáneamente varias conductas han sido recomendadas siguiendo criterios de eficiencia ${ }^{9}$ $\mathrm{y}$ han demostrado ser eficaces ${ }^{10}$. Por otro lado, se debería propugnar una Educación para la Salud (EpS) transversal ${ }^{11}$ en el ámbito escolar, ya que uno de los objetivos de la educación secundaria es valorar críticamente la salud y los hábitos sociales relacionados con ella ${ }^{12}$. Por esta razón, la escuela es un contexto preferencial para implementar intervenciones educativas destinadas al control de las conductas de riesgo de cáncer.

Internet y la telefonía móvil forman parte de la tecnología más reciente que puede utilizarse para EpS. En España, el 99,7\% de los centros de enseñanza secundaria están informatizados y tienen conexión a Internet ${ }^{13}$. En México el porcentaje es menor, pero la penetrancia de la telefonía móvil en el colectivo de adolescentes es muy elevada en ambos países, por lo que se dan las condiciones adecuadas para utilizar estas tecnologías como instrumento de la EpS. En la literatura científica existen ya muchas experiencias sobre su utilización para generar conductas saludables ${ }^{14-17}$, pero normalmente su impacto se evalúa en situaciones muy controladas. Sería deseable conocer, por tanto, cuál es el interés que podría suscitar en la población diana una intervención que utilice internet y la telefonía móvil en condiciones reales de aplicación. Además, también podría ser útil comparar su desarrollo en dos países con realidades educativas, culturales y tecnológicas diferentes.

Evaluar el proceso supone constatar si un programa ha alcanzado a la población diana y si se han ejecutado adecuadamente las actividades planificadas ${ }^{18}$. Por las características de nuestro programa, el programa Prevencanadol, las actividades debían estar ya ejecutadas antes de la implementación propiamente dicha, ya que era el estudiante el responsable de elegir cuándo y cómo se auto-administraba las actividades que formaban la intervención. Por esta razón, este artículo está centrado en el alcance, aunque también en otros aspectos importantes de la evaluación de programas en el medio escolar, como la magnitud de la exposición y el uso continuado de las actividades a través del tiempo ${ }^{19}$.

El objetivo fue evaluar el proceso de un programa para prevenir conductas de riesgo de cáncer implementado a través de internet y telefonía móvil. 


\section{SUJETOS Y MÉTODOS}

Tipo de estudio. Estudio evaluativo del proceso de implementación del programa Prevencanadol (acrónimo de prevención del cáncer en adolescentes).

Descripción del programa. Se realizó un ensayo clínico controlado y aleatorizado que evaluó la eficacia de una intervención educativa basada en la Web y en la telefonía móvil para prevenir, y en su caso modificar, las conductas de riesgo en adolescentes y en su entorno. La metodología se ha publicado en otro manuscrito ${ }^{20}$. En síntesis, se diseñó una página web interactiva (www.alertagrumete.com y su versión homóloga mexicana (www.alertagrumete.com.mx), que contó con elementos de la Web 2.0, de las actuales redes sociales (figura 1) y que estuvo basada en el currículo escolar. Durante los cursos académicos 2009-10, 2010-11 y 201112 los profesores de enseñanza secundaria interesados invitaron a sus alumnos de $1^{\circ}$ a $3^{\circ}$ de educación secundaria (12 a 16 años) a registrarse en la web del programa. El rol desempeñado por el profesorado fue libremente elegido por cada uno: unos solamente dieron a conocer el programa entre los estudiantes, otros dedicaron horas de clase a las actividades del programa y otros no juzgaron interesante o prioritaria la iniciativa y, por tanto, no intervinieron. El registro se realizó mediante usuario y contraseña para que los accesos fueran individuales, anónimos y controlados. Los estudiantes que decidieron participar fueron asignados aleatoriamente a un grupo experimental (GE) o a un grupo control (GC). Únicamente los estudiantes del GE tuvieron acceso a la página web durante el curso académico que duró su intervención.

Participar en el programa Prevencanadol suponía resolver problemas dirigidos a mejorar las competencias específicas del

\section{Figura 1}

\section{Página principal y secciones de la página web del programa Prevencanadol}

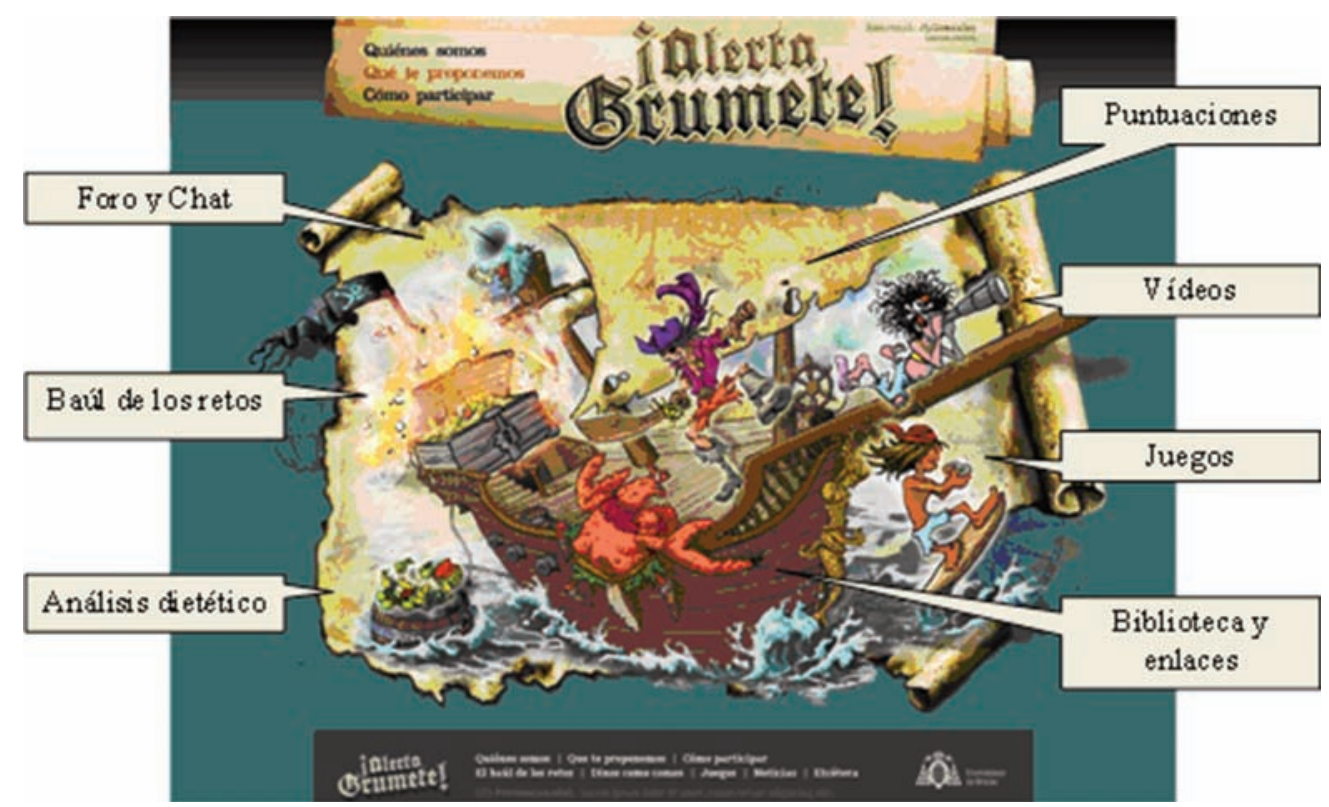


currículo escolar (matemática, lingüística, social y ciudadana, etc.), pero también relacionados con la prevención de conductas de riesgo. Por ejemplo, el enunciado de uno de estos problemas dirigido específicamente a mejorar la competencia matemática decía: "Si en México mueren aproximadamente 56.350 personas por causa del cáncer anualmente y se estima que el tabaco origina el $30 \%$ del total de casos ¿cuántas muertes se podrían evitar al cabo de 5 años, si todos los mexicanos dejaran de fumar?". Además, los estudiantes del GE tenían libre acceso a: (a) vídeos educativos de corta duración grabados por otros estudiantes; (b) foros de discusión y chats moderados sobre las desventajas de practicar conductas de riesgo; (c) envío de recetas y recuerdos de ingestas de las 24 horas previas para recibir consejo experto sobre la dieta; (d) abundante información y (e) juegos educativos, etc. (figura 1). Adicionalmente, los estudiantes con teléfono móvil recibían semanalmente mensajes (SMS) de carácter motivador para seguir los consejos preventivos del CECC.

Para alentar la participación, el programa se configuró como un concurso, de forma que realizar las actividades descritas y visitar la web proporcionaba puntos a cada estudiante. Finalizar el curso académico entre las 20 primeras posiciones del ranking daba opción a elegir un regalo.

Un requisito obligatorio para los estudiantes del GE y los del GC fue realizar un cuestionario inicial y otro final, que requerían entre 25 y 30 minutos para ser cumplimentados. Por otro lado, los estudiantes del GE podían invitar a personas adultas a participar en el programa con el objetivo secundario de comprobar si influían positivamente en ellas y si mejoraban también su adherencia a los consejos del CECC.

El programa cumplió los principios establecidos en la Declaración de Helsinki. Específicamente, todos los participantes dieron consentimiento informado y contó con la aprobación del Comité Institucional de Ética. Ade- más, el programa se incluyó en un registro internacional de ensayos clínicos aceptados por la OMS (ISRCTN27988779).

Difusión del programa. Durante la fase de diseño, el programa se presentó en la $8^{\mathrm{a}}$ Conferencia Anual de la Asociación Americana para la Investigación sobre el Cáncer ${ }^{21}$ y en la 20 a Conferencia Mundial de Promoción de la Salud ${ }^{22}$. Además, el esfuerzo en su difusión fue máximo entre los profesores de secundaria, que eran los principales encargados de animar a participar al alumnado si juzgaban interesante el programa. Específicamente: (a) se publicitó en medios españoles de comunicación de masas (prensa, revistas y radio); (b) se colocó el enlace a www.alertagrumete.com en las principales páginas web institucionales del sistema educativo; (c) se enviaron correos electrónicos a todos los profesores de educación secundaria de España y México; y (d) se presentó la iniciativa e instruyó sobre el manejo del recurso online mediante videoconferencia a los profesores interesados. El control sobre estas estrategias de difusión pudo ser más efectivo en las regiones de filiación de los creadores del programa Prevencanadol (Asturias en España y Nuevo México en México). También se comprobó que el programa satisfacía la mayoría de requisitos de aceptación de los que existe evidencia ${ }^{23,24}$ : interfaz atractiva, navegación sencilla, recuerdos para fomentar las visitas, retroalimentación periódica, actividades interactivas adaptadas a la edad, incentivar la competición y posibilitar recompensas, etc.

Fuente de información y análisis estadístico. Debido a las especiales características de la página web fue necesario crear una plataforma de gestión específica (www.alertagrumete.com/backend.php). Además de utilizarse como soporte para la gestión privada de la página web, permitió controlar anónimamente a los usuarios durante las fases de implementación: (a) fase de registro (reclutamiento) y (b) fase de participación (adherencia). Para obtener in- 
formación adicional sobre las variables previamente mencionadas también fue necesario recurrir al servicio Web Google Analytics, aplicación que, entre otras posibilidades, permite obtener informes de seguimiento de los usuarios de las páginas web y realizar análisis de navegación. De esta manera se obtuvo información de la forma de acceso a la página web (tipo de navegador, motor de búsqueda, acceso procedentes de otras páginas, palabras clave, etc.), disposición de tecnología flash para reproducir las animaciones multimedia, rebote (porcentaje de accesos únicos a la página web sin realizar ninguna acción) y se comparó con otras páginas web similares utilizando diferentes indicadores (tráfico medio, categoría salud y subcategoría educación sanitaria y entrenamiento médico). Asimismo se tuvieron en cuenta otras variables sociodemográficas y académicas básicas expuestas más adelante.

La información se recuperó en formato Microsoft Excel para Windows y posteriormente se transformó en una base de datos del programa SPSS v.19.0 (SPSS Inc., Chicago, Illinois). Se midieron los indicadores de reclutamiento y de la adherencia que figuran a continuación y se compararon según el curso académico de implementación (1: 2009-10, 2: 2010-11 y 3: 2011-12):

a) Reclutamiento: número de usuarios y características demográficas y académicas básicas (edad, sexo, estudios del padre y de la madre, disponibilidad de teléfono móvil, curso escolar y nivel académico), forma de acceso (tipo de navegador, motor de búsqueda y palabras clave), lugar (país y región) y disponibilidad de teléfono móvil.

b) Adherencia: número de accesos y duración, número y tipo de secciones visitadas por acceso, porcentaje de rebote, duración de la intervención, número de adultos invitados (los adultos podían participar de forma espontánea o merced a la invitación de un adolescente; en este caso, durante el registro debían especificar el alias del usuario adolescente que les había invitado y su relación) y tasa de retención del programa (porcentaje de estudiantes que contestaron el cuestionario post-intervención).

En general, los análisis se realizaron de forma estratificada distinguiendo entre la implementación en España (cursos 1 y 2) y en México (curso 3) y únicamente fueron considerados estadísticamente significativos los resultados con $p<0,05$. Se llevaron a cabo la obtención de medias y sus intervalos de confianza al $95 \%$ (IC95\%), la distribución de frecuencias absolutas y relativas (\%) y la realización de contrastes a nivel bivariante para buscar diferencias entre los distintos períodos de implementación $\left(\chi^{2}\right.$ de Pearson, $t$ de Student, ANOVA seguido del test de Tukey). También se obtuvieron los coeficientes $\beta$ de regresión lineal para la asociación entre el número de accesos y su duración y el número de días que duró la implementación. Por último, se realizó un análisis exploratorio mediante regresión logística para calcular las odds ratio (OR) ajustadas de las características de los estudiantes del GE retenidos en el programa.

\section{RESULTADOS}

Reclutamiento. Iniciaron el registro en la web 3.855 estudiantes y lo completaron $2.001(51,9 \%)$ de los que $1.550(77,5 \%)$ fueron captados durante el curso académico de ejecución del programa en México $(\mathrm{p}<0,001)$ (figura 2). Otras características de los participantes se pueden ver en la tabla 1.

El navegador preferido por los usuarios de las páginas web del programa fue Internet Explorer, pero el su uso decreció del curso académico 1 al 3 del $65,5 \%$ al $32,5 \%$ a favor de otras aplicaciones más recientes como Google Chrome, que experimentó la tendencia contraria (de $12,2 \%$ en el curso 1 a 59,7\% durante el 3). La utilización de telé- 
Figura 2

Diagrama del flujo del reclutamiento y distribución a los grupos del estudio

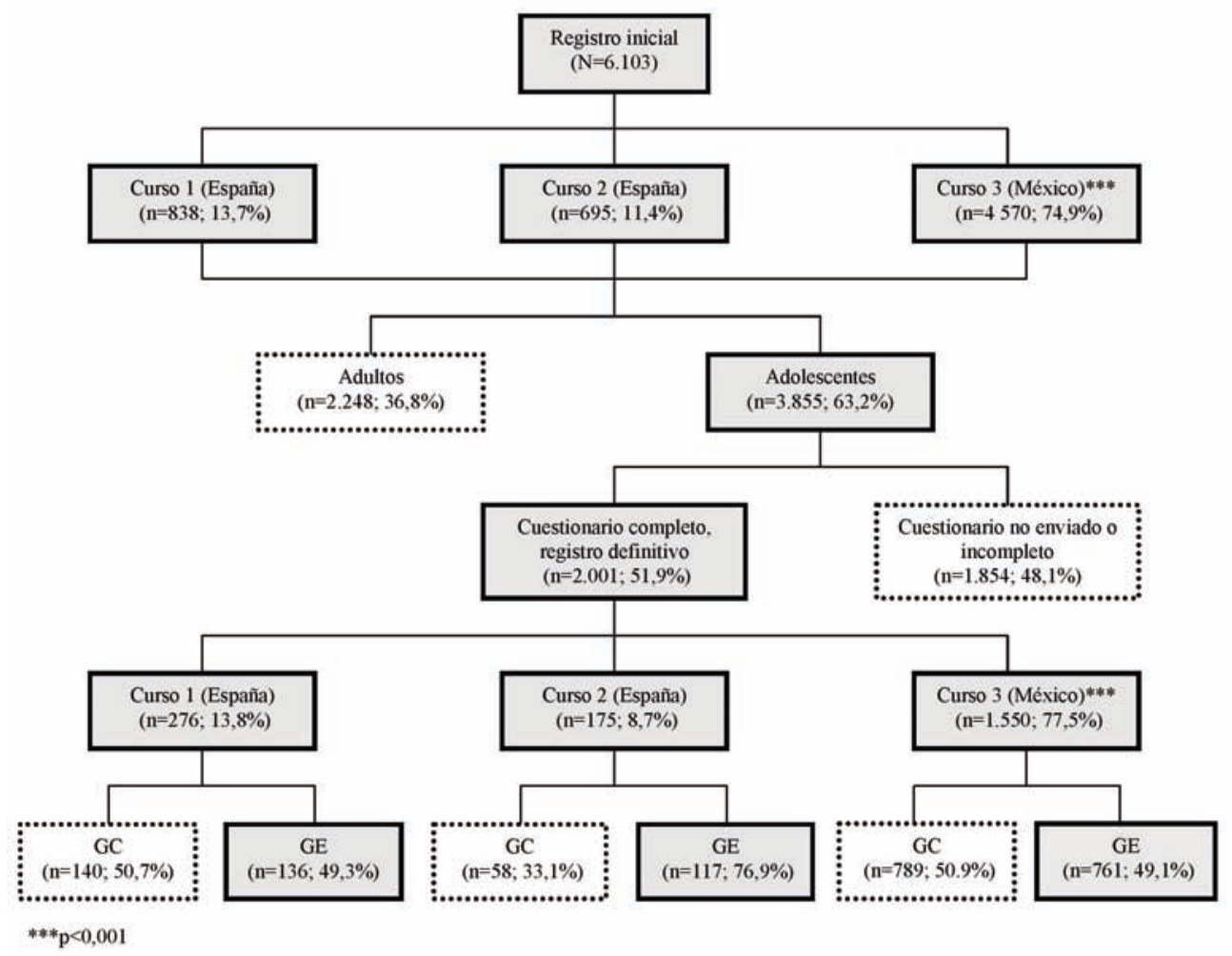

fonos móviles inteligentes (smartphones) para acceder a la web creció un $100 \%$, aunque su frecuencia de uso en el último curso académico fue del 0,8\%. El motor de búsqueda Google se utilizó en el 91,1\%, aunque sufrió un decrecimiento del $-5,4 \%$ desde el curso académico 1 al 3 .

Durante la implementación del programa en España, la forma más frecuente de acceder a la web fue insertar palabras clave, como "alerta grumete" o "alertagrumete", en un motor de búsqueda $(55,3 \%$ en el curso 1 y $75,8 \%$ en el 2). Sin embargo, en México en el 38,4\% de los casos escribieron directamente la dirección www.alertagrumete.com.mx en la barra de navegación y en $34,1 \%$ hicieron clic sobre los enlaces alojados en otras páginas web. Entre los enlaces más habituales, aparecen en se- gundo lugar los localizados en páginas de la red social Facebook $(9,3 \%)$ y a continuación los insertados en otras similares como YouTube o Blogger $(4,4 \%)$.

El 98,6\% de los usuarios dispuso de la versión flash requerida para visionar las animaciones de la web. El 1,4\% no, aunque podían visitar igualmente las secciones. El porcentaje fue superior durante el curso académico de implementación en México que durante los de ejecución en España $(0,8 \%$ vs. $0,3 \% ; p<0,001)$. Esta circunstancia también afectó a quienes accedieron mediante smartphones $(0,6 \%)$.

En los tres cursos académicos se produjeron accesos a las web española y mexicana desde países de todo el mundo, sobre todo de países donde el español es lengua oficial. No obstante, 
Tabla 1

Características sociodemográficas y escolares básicas de los participantes según curso académico y grupo de estudio

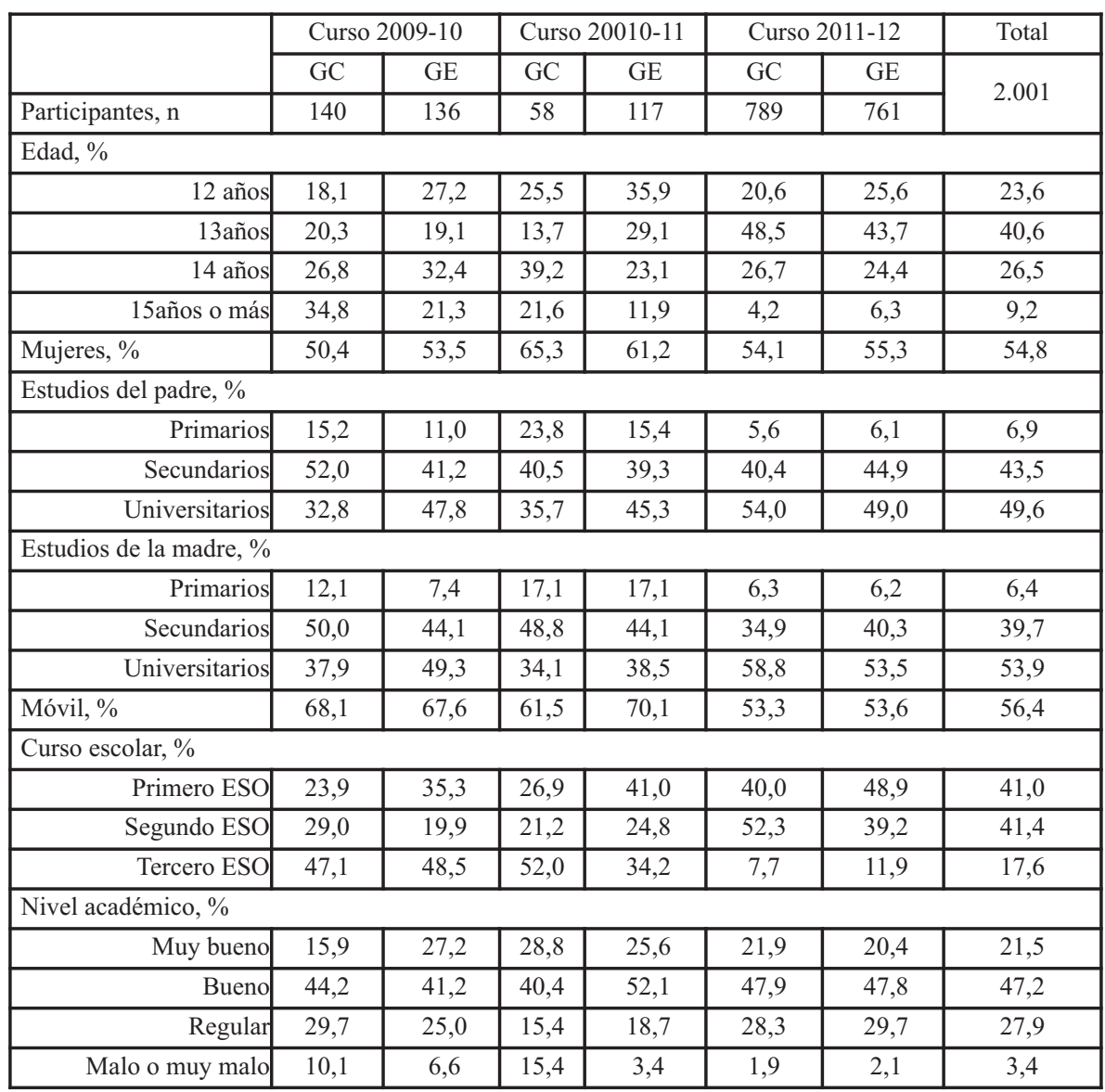

GC: Grupo de Control. GE: Grupo Experimental. ESO: Educación Secundaria Obligatoria

el $97,9 \%$ de los accesos durante el curso 1 y el $96,7 \%$ durante el 2 se produjeron desde España, y el 97,0\% de los accesos durante el curso 3 se produjeron desde México. De igual manera, dentro de cada país, el porcentaje más elevado de visitas provino de equipos informáticos situados en las regiones donde debutó el programa: Asturias (83,9\% de las visitas en el curso 1 y $78,8 \%$ en el curso 2) y Nuevo León (96,5\% durante el curso 3).

El $56,5 \%$ de los estudiantes registró un número de teléfono móvil $\mathrm{y}$, por tanto, recibieron una intervención educativa completa: participación en la Web y recepción de SMS educativos. Este porcentaje fue del $67,1 \%$ España y del $53,4 \%$ en México $(\mathrm{p}<0,001)$.

Adherencia. De los 2.001 participantes totales en el programa, fueron objeto del análisis de adherencia los 1.014 adolescentes asignados al GE, ya que fue el que utilizó la web como recurso educativo y tenía privilegios de acceso a todas las secciones. 
Al finalizar el período de implementación (2009-12), el número de accesos contabilizados fue 51.850 que se distribuyeron según los datos de la tabla 2. Como se puede observar, la media de visitas diarias a la web mexicana fue de 186,6: IC95\%: 164,0$209,3)$ superior a la media de españa durante el curso $1(36,4$ : IC95\%: $31,0-37,9)$ y el curso 2 (24,4; IC95\%: 22,8-26,0). La duración media de las visitas (186,6; IC95\%: 164,0-209,3) también fue superior en el curso académico 3 .

En los tres cursos académicos, a medida que pasaron los días de ejecución del programa, disminuyó el número de visitas (figura 3a). El coeficiente de regresión indica que la relación negativa fue más acusada durante la implementación en México ( $\beta 3=$ $0,43 ; \mathrm{p}<0,001)$ que en los cursos académicos españoles $(\beta 1=-0,15 ; p<0,01$ y $\beta 2=$ $0,34 ; \mathrm{p}<0,001)$. De la misma manera, se estudió la evolución del tiempo medio de duración del acceso en minutos (figura 3b) que reveló que, mientras que en el curso académico 1 aumentó con los días de ejecución $(\beta 1=0,52 ; p<0,001)$ en los otros dos cursos académicos sucedió lo contrario $(\beta 2=-0,19 ; p<0,01$ y $\beta 2=-0,22 ; p<0,01)$.

Durante el curso académico 1, cada individuo accedió a la Web en 31,6 ocasiones y visitó una media de 17,2 secciones en cada acceso. Durante el curso académico 2 cada usuario accedió 21,8 veces y visitó 11,8 secciones y, durante el curso académico 3, hubo también 21,9 accesos por usuario que visitó una media de 13,0 secciones. Invariablemente, durante los tres cursos académicos más del 50\% de los usuarios del GE visitaban al menos 5 secciones de la página web $(74,4 \%$ en el curso $1 ; 63,7 \%$ en el curso 2 y $59,0 \%$ en el curso 3 ). Las secciones más visitadas durante la implementación en España fueron el "ranking de puntuaciones" $(5,2 \%$ del total en el curso 1 y $4,3 \%$ en el curso 2) y el "baúl de los retos" $(4,7 \%$ y $5,9 \%$, respectivamente). Por otro lado, los estudiantes mexicanos visitaron más la sección que explica "cómo participar" $(4,6 \%)$ seguida del "baúl de los retos" $(4,3 \%)$. La sección de "juegos" no se encontró en ningún curso académico entre las 10 más visitadas.

Tabla 2

\section{Descripción del número de visitas al programay de su duración según curso académico}

\begin{tabular}{|l|l|c|c|}
\hline Curso académico & & Visitas & Tiempo (horas:minutos:segundos) \\
\hline \multirow{5}{*}{ Curso 2009-10 } & Media/día IC95\%) & $34,4(31,0-37,9)$ & $00: 11: 02(00: 10: 24-00: 11: 40)$ \\
\cline { 2 - 4 } & Mínimo/día & 0,0 & $00: 00: 00$ \\
\cline { 2 - 4 } & Máximo/día & 254,0 & $00: 31: 37$ \\
\cline { 2 - 4 } & Total & 10.462 & $07: 55: 45$ \\
\hline \multirow{5}{*}{ Curso 2010-12 } & Media/día (IC95\%) & $24,4(22,8-26,0)$ & $00: 08: 07(00: 07: 34-00: 08: 40)$ \\
\cline { 2 - 4 } & Mínimo/día & 0,0 & $00: 00: 00$ \\
\cline { 2 - 4 } & Máximo/día & 86,0 & $00: 34: 17$ \\
\cline { 2 - 4 } & Total & 7.421 & $17: 05: 57$ \\
\hline \multirow{5}{*}{ Curso 2011-12 } & Media/día IC95\%) & $186,6(164,0-209,3)^{* * *}$ & $00: 12: 55(00: 12: 24-00: 13: 25)^{* * * *}$ \\
\cline { 2 - 4 } & Mínimo/día & 2,0 & $00: 01: 05$ \\
\cline { 2 - 4 } & Máximo/día & 692,0 & $00: 23: 12$ \\
\cline { 2 - 4 } & Total & 33.967 & $15: 10: 24$ \\
\hline
\end{tabular}

$* * * \mathrm{p}<0,001$ 
Figura 3

Evolución del número de visitas diarias al programa (3a) y de su duración (3b) según curso académico (1: 2009-10; 2: 2010-11; 3: 2011-12)

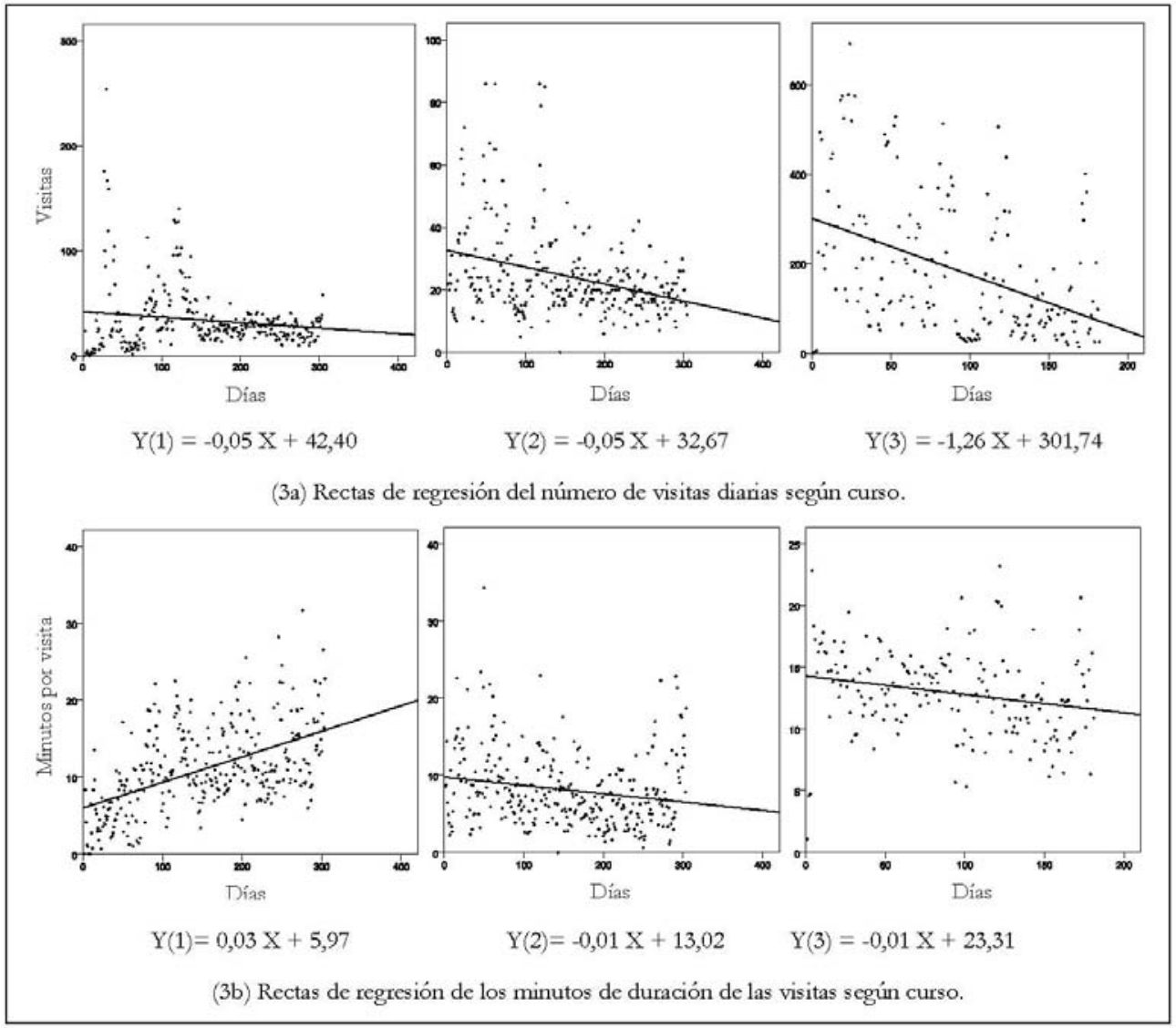

El porcentaje de rebote tuvo diferencias estadísticamente significativas $(\mathrm{p}<0,001)$ en los diferentes cursos académicos: $14,8 \%$ (IC95\%:13,5-16,3) en el curso 1; 29,6\% (IC95\%:27,9-31,4) en el curso 2 y 34,1\% (IC95\%:33,1-35,2) en el curso 3 lo que no se asoció con variables tecnológicas, como poseer la versión flash requerida.

Durante su participación, los estudiantes del GE invitaron y consiguieron el registro en la página Web de 1.172 adultos de su entorno. En el curso académico 1, cada adolescente logró enrolar a una media de 1,06 adultos (IC95\%: 1,01-1,09), en el curso aca- démico 2 esta media fue 0,81 (IC95\%: 0,74$0,88)$ y en el curso académico 3 fue 1,23 (IC95\%: 1,14-1,29).

La duración media de la implementación en los tres cursos académicos fue, consecutivamente: 201,5 días (IC95\%: 195,7207,4), 270,7 días (IC95\%: 259,7-281,6) y 144,4 días (IC95\%:142,1-146,7). Por último, contestaron el cuestionario final 421 estudiantes del GE, por lo que la tasa de retención global del programa fue $41,5 \%$. Las características de los estudiantes del GE que mejor explicaron la probabilidad de ser retenido por el programa fueron: tener nivel 
académico muy bueno (OR: 12,5; IC95\%: 1,6-88,6) o bueno (OR: 9,6; IC95\%: 1,264,6), ser mexicano (OR: 4,4; IC95\%: 2,67,6), tener 12 años (OR: 3,1; IC95\%: 1,2$8,1)$ y haber realizado el registro en la web durante el primer trimestre del curso académico (OR: 2,8; IC95\%:2,0-3,9).

\section{DISCUSIÓN}

El reclutamiento de participantes fue escaso ya que iniciaron el registro 3.855 estudiantes, cuando solo la población de 12 a 16 años de Nuevo León y Asturias ronda el medio millón ${ }^{25,26}$. Además, hubo un gran número de adolescentes que iniciaron el registro pero no completaron el cuestionario obligatorio para continuar en el programa. Como señalan Nebot et al ${ }^{18}$, alcanzar el equilibrio entre el rigor metodológico y otras cuestiones que pueden afectar a la factibilidad del programa es tan necesario como difícil. En nuestro programa, es posible que las mediciones necesarias para evaluar una intervención tan compleja fueran demasiado exhaustivas y el tiempo exigido para contestar excesivo. Es cierto que los adolescentes prefieren cuestionarios onli$n \mathrm{e}^{23}$, como el del programa Prevencanadol, pero además que sean cortos y sencillos. En este sentido, los expertos coinciden en que la extensión y tiempo de los cuestionarios son quizá los condicionantes más importantes del éxito del reclutamiento ${ }^{24}$. Aun así, el número de participantes fue superior al de intervenciones similares ${ }^{27,28}$ y suficiente para alcanzar los objetivos de la investigación, uno de los cuáles fue la evaluación del proceso de implementación que ahora presentamos. En un metanálisis sobre programas online, Webb et al ${ }^{29}$ analizaron 85 artículos en los que la media de usuarios registrados fue aproximadamente de 500, notablemente inferior a los de nuestro programa.

El análisis de las características sociodemográficas y académicas básicas revelaron que este tipo de iniciativas pueden ser ligeramente más apreciadas por las adolescen- tes, aun cuando la estética de la página principal del dominio www.alertagrumete.com, a priori, nos hacía pensar que podría ser más atractiva para los varones. Este es un hallazgo compartido por otros estudios, ya que las mujeres son usuarias más habituales de páginas web relacionadas con la salud, sobre todo si abordan cuestiones acerca del control del peso o la alimentación ${ }^{28,30}$. También es llamativo el elevado nivel de instrucción paterno, que quizá refleje que los estudios universitarios proporcionan mayor sensibilidad hacia estos programas y, por tanto, mayor tendencia a animar a los hijos a participar en ellos. Por otro lado, no es extraño que este tipo de iniciativas involucre a adolescentes con mejor nivel académico, ya que esta es una variable que determina muchas conductas de salud, como ser abstemio o realizar ejercicio físico ${ }^{31,32}$.

El reclutamiento fue máximo en Asturias (España) y Nuevo León (México) y anecdótico en otros lugares. Teniendo en cuenta que la mayoría de programas educativos dirigidos a población sana tiene problemas en esta fase ${ }^{33}$, quizá hubiera sido preferible apostar por una difusión local porque, en la práctica, sigue siendo más sencillo y barato intervenir sobre un entorno homogéneo que facilite el control.

Existen interesantes herramientas para realizar evaluaciones del proceso de forma protocolizada. Por ejemplo, Ariza et al ${ }^{19}$ sugieren tener en cuenta ocho estándares mínimos, de los cuales el programa Prevencanadol solamente incumple el referido a alcanzar una tasa de seguimiento igual o superior al $80 \%$. Por otro lado, el sistema RE-AIM propone evaluar las dimensiones de las siglas que forman su nombre (del inglés Reach, Effectiveness, Adoption, Implementation y Manteinance) con una serie de ítems sencillos ${ }^{34}$. Parece razonable pensar que todas las dimensiones, excepto una, fueron satisfechas: (a) el programa fue conocido por muchos estudiantes (Reach=alcance); (b) se utilizaron múltiples 
variables de respuesta que permiten comparaciones (Effectiveness=efectividad), si bien la evaluación de la eficacia no es el objetivo de este artículo; (c) se realizaron todas las actividades programadas, que además fueron adaptadas a la cultura mexicana (Implementation=implementación); y (d) las mediciones pudieron ser realizadas al finalizar la intervención (Manteniance $=$ mantenimiento). No obstante, el escaso número de estudiantes que participó hace pensar que el porcentaje de centros educativos captados también fue inferior al esperado (Adoption=adopción), quizá por la falta de motivación del profesorado. Por otro lado, el reclutamiento fue más satisfactorio en México, circunstancia que podría deberse a una mejor conservación de la autoridad del profesor en el país americano. Este argumento lo podría sustentar el hecho de que en México los adolescentes accedieran más frecuentemente escribiendo la dirección www.alertagrumete.com.mx en la barra de direcciones, lo que sugiere un acceso más dirigido. Pero también podría obedecer simplemente a un mayor interés, por ser en este país un enfoque más novedoso.

Habida cuenta de la rutina de los accesos y su evolución, los futuros programas preventivos en línea deben intentar adaptarse a todos los navegadores y motores de búsqueda existentes, ya que anticipar durante su diseño cuál será el más utilizado es difícil por el acelerado ritmo evolutivo de Internet. Lo que parece claro es que la tendencia actual lleva hacia la utilización de smartphones y redes sociales ${ }^{35,36}$, lo que también queda demostrado en nuestro estudio con la evolución de los accesos a la web.

Otras diferencias en el proceso de reclutamiento de participantes entre los dos países se produjeron en el porcentaje de usuarios que tenía una versión flash demasiado antigua para soportar las animaciones de la web y en el de usuarios con teléfono móvil. En ambos casos, los estudiantes mexicanos carecían con mayor frecuencia de las herra- mientas más apropiadas, por lo que se pone de manifiesto que cuando se utilizan las tecnologías de la comunicación actuales es más necesario que nunca adaptar las intervenciones a los recursos de la población diana, sobre todo si queremos llegar a los grupos socialmente más desfavorecidos, en los que se suelen combinar menores recursos tecnológicos y mayor prevalencia de conductas de riesgo.

Las visitas diarias guardan una estrecha y evidente relación con el número de usuarios reclutados, de forma que es lógico que durante el curso académico 3 fueran más frecuentes que durante el resto de cursos porque también hubo más participantes mexicanos que españoles. La mayor duración media de las visitas en México (entre 2 y 5 minutos más) podría deberse, una vez más, a que este tipo de innovaciones docentes son menos frecuentes y más apreciadas. Por otro lado, procede destacar que los recursos educativos capaces de captar la atención de los usuarios más de 10 minutos son los que pueden tener impacto significativo sobre las conductas ${ }^{37}$. En nuestro estudio la duración únicamente fue inferior en el curso académico 2 y no parece suficientemente amplia para influir demasiado en el potencial impacto de la intervención, lo que se constatará o no cuando llevemos a cabo la evaluación de la eficacia. En cualquier caso, prácticamente en todos los cursos académicos, tanto el número de visitas como su duración disminuyeron a medida que avanzaba la ejecución, lo que puede traducir una pérdida de interés en las actividades propuestas. Sería interesante que los programas preventivos del futuro lograran conciliar el tiempo durante el que se mantiene el interés en participar con el necesario para conseguir cambios conductuales.

El análisis de las secciones más visitadas reveló que la sección con mayor potencial educativo, el "baúl de los retos", fue muy popular y que, sin embargo, la sección de ocio tuvo menos éxito, quizá porque lo 
realmente motivador para los adolescentes fue la competencia generada con los ejercicios planteados. Este hallazgo es interesante porque, aunque los juegos interactivos son muy atractivos para niños y adolescentes ${ }^{38}$, parece que lo realmente interesante para mejorar la adherencia es fomentar la competitividad, sea cual sea la actividad propuesta. En México fue más habitual visitar la sección donde figuraban las instrucciones para la participación, lo que nuevamente evoca una mayor novedad de estas herramientas en el país norteamericano y confirma la conveniencia de ofrecer explicaciones operativas muy detalladas y sencillas cuando la población diana no está familiarizada con este tipo de recursos.

El porcentaje de rebote, considerado un indicador indirecto del acceso por error, fue muy satisfactorio si se compara con el $45,2 \%$ que tienen de media páginas web similares (tráfico medio, categoría salud y subcategoría educación sanitaria y entrenamiento médico, según informe de Google Analytics) $)^{39}$ y que fue utilizado por nosotros como referencia ${ }^{40}$.

La tasa de retención de nuestro programa fue modesta. En cualquier caso, los programas de intervención para cambiar conductas de riesgo en adolescentes voluntarios suelen tener tasas de retención similares. El programa HELENA ${ }^{41}$, implementado en 6 países europeos, obtuvo tasas de retención sólo ligeramente superiores, pero su evaluación fue realizada a los 3 meses y la de nuestro estudio después de cada curso académico completo. En el mencionado programa los participantes no se diferenciaron de los no participantes ni en sexo ni en edad ${ }^{42}$. Sin embargo, nuestro programa fue más aceptado por los estudiantes más jóvenes, circunstancia que invita a pensar que, cuando se trabaja con niños y adolescentes, es conveniente estrechar el rango de edad para maximizar la retención. Como en el nuestro, otros estudios también encuentran que los progra- mas son mejor tolerados por los sujetos que tienen mejor nivel académico, sobre todo si juzgan que los contenidos del programa les pueden ser útiles ${ }^{43}$.

En resumen, el reclutamiento de participantes fue escaso, fundamentalmente en España y entre los adolescentes con peor rendimiento académico y procedentes de familias con bajo nivel de instrucción. Los indicadores de adherencia fueron aceptables entre los participantes que además son capaces de reclutar un elevado número de adultos de su entorno, no obstante se observó pérdida de interés en las actividades propuestas $\mathrm{y}$, finalmente, el programa perdió un elevado porcentaje de estudiantes.

Los investigadores que deseen implementar proyectos análogos deberían cumplir los requisitos de efectividad identificados por otros estudios, difundirlo local o regionalmente para ser más eficientes, simplificar los cuestionarios y la duración de las intervenciones hasta lo metodológicamente asumible, utilizar la tecnología más puntera, atender a las particularidades de las poblaciones con menor nivel de desarrollo y adaptar la herramienta a sus recursos (quizá más obsoletos) y necesidades (mayor necesidad de instrucciones).

\section{AGRADECIMIENTOS}

Los autores agradecen especialmente la contribución del Profesor Hein de Vries, Catedrático de la Universidad de Maastricht, y la de todos los profesores que ayudaron a adaptar la página web al currículo escolar mexicano y español y a implementar el programa en sus aulas. También agradecen la inestimable colaboración de la profesora Goretti Faya Ornia como asesora lingüística y traductora. Y, por supuesto, recuerdan con cariño al profesor Antonio Cueto Espinar, colaborador en la investigación, quien tristemente falleció sin verla terminada. 


\section{BIBLIOGRAFÍA}

1. Sánchez MJ, Payer T, De Angelis R, Larrañaga N, Capocaccia R, Martinez C, et al. Cancer incidence and mortality in Spain: estimates and projections for the period 1981-2012. Ann Oncol. 2010; 21:30-36.

2. Cabanes A, Vidal E, Aragonés N, Pérez-Gómez B, Pollán M, Lope V, et al. Cancer mortality trends in Spain: 1980-2007. Ann Oncol. 2010; 21:14-20.

3. Ferlay J, Shin HR, Bray F, Forman D, Mathers C, Parkin DM. GLOBOCAN 2008 v1.2, Cancer Incidence and Mortality Worldwide: IARC CancerBase No. 10 [base de datos en Internet]. Lyon: International Agency for Research on Cancer; 2010 [citado 2013 mayo 19]. Disponible en: http://globocan.iarc.fr.

4. Siegel R, Naishadham D, Jemal A. Cancer statistics, 2012. CA Cancer J Clin. 2012; 62:10-29.

5. Eaton DK, Kann L, Kinchen S, Shanklin S, Flint $\mathrm{KH}$, Hawkins J, et al. Youth risk behavior surveillance - United States, 2011. MMWR Surveill Summ. 2012; 61:1-162.

6. Association of European Cancer Leagues [Internet]. Bruselas: Association of European Cancer Leagues; 2010 [actualizado 2013 febrero 01; citado 2013 junio 11]. Disponible en: http://www.europeancancerleagues.org/index.php

7. Secretaría de Salud (Subsecretaría de Prevención y Promoción de la Salud). Programa de acción específico 2007-2012, Escuela y Salud. 2a edición. México DF: Secretaría de Salud; 2009.p.41.

8. Instituto de Salud Carlos III. La situación del cáncer en España. Madrid: Ministerio de Sanidad y Consumo;2009.p.113-114.

9. Prochaska JJ, Nigg CR, Spring B, Velicer WF, Prochaska JO. The benefits and challenges of multiple health behavior change in research and in practice. Prev Med. 2010; 50:26-29.

10. López ML, Iglesias JM, del Valle MO, Comas A, Fernández JM, de Vries H, et al. Impact of a primary care intervention on smoking, drinking, diet, weight, sun exposure, and work risk in families with cancer experience. Cancer Causes Control. 2007; 18:525535.

11. Lee A. Health-promoting schools: evidence for a holistic approach to promoting health and improving health literacy. Appl Health Econ Health Policy. 2009; 7:11-17.
12. Boletín Oficial del EstadoLey Orgánica de Educación. Ley Orgánica 2/2006, de 3 de mayo de Educación. BOE núm 106 de 04-06-2006.

13. Instituto de Tecnologías Educativas. Indicadores y datos de las tecnologías de la información y comunicación en la educación en Europa y España [Internet]. Madrid: Ministerio de Educación;2011 [citado 2013 mayo 13 ]. Disponible en: http://recursostic.educacion.es/blogs/europa/media/ blogs/europa/informes/indicadores_y_datos_tic_eu ropa_y_espa_a_09_10_ite_.pdf

14. Rooke S, Thorsteinsson E, Karpin A, Copeland J, Allsop D. Computer-delivered interventions for alcohol and tobacco use: a meta-analysis. Addiction. 2010; 105:13811390 .

15. Hamel LM, Robbins LB, Wilbur J. Computer- and web-based interventions to increase preadolescent and adolescent physical activity: a systematic review. J Adv Nurs. 2011; 67:251-268.

16. Andersson G, Ljótsson B, Weise C. Internet-delivered treatment to promote health. Curr Opin Psychiatry. 2011; 24:168-172

17. Nguyen B, Kornman KP, Baur LA. A review of electronic interventions for prevention and treatment of overweight and obesity in young people. Obes Rev. 2011; 12:e298-314.

18. Nebot M, López MJ, Ariza C, Villalbí JR, GarcíaAltés A. Evaluación de la efectividad en salud pública: fundamentos conceptuales y metodológicos. Gac Sanit. 2011;25:S3-8.

19. Ariza C, Villalbí JR, Sánchez-Martínez F, Nebot M. La evaluación del proceso en relación con la evaluación de la efectividad: experiencias de programas en el medio escolar. Gac Sanit. 2011;25:S32-39.

20. Lana A, Del Valle MO, López S, Faya-Ornia G, López ML. Study protocol of a randomized controlled trial to improve cancer prevention behaviors in adolescents and adults using a web-based intervention supplemented with SMS. BMC Public Health. 2013; 13:357.

21. López ML, Lana A. Multiple behavior interventions among cancer survivors and their relatives. Cancer Prev Res. 2010; 3:CN09-04.

22. Lana A, Cueto A, Secall R, van Riet E, López ML. A Web-based intervention to promote cancer prevention through multiple behaviour change among adolescents and their teachers. Libro de ponencias de la 20th IUPHE World Conference on Health Promotion; 2010 11-15-jul: Geneva: International Union for Health Promotion and Education; 2010. 
23. de Nooijer J, Veling ML, Ton A, de Vries H, de Vries NK. Electronic monitoring and health promotion: an evaluation of the E-MOVO Web site by adolescents. Health Educ Res. 2008; 23:382-391.

24. Crutzen R, de Nooijer J, Brouwer W, Oenema A, Brug $\mathrm{J}$, et al. Internet-delivered interventions aimed at adolescents: a Delphi study on dissemination and exposure. Health Educ Res. 2008; 23:427-439.

25. Instituto Nacional de Estadística. Explotación estadística del padrón [base de datos en Internet]. Madrid: Instituto Nacional de Estadística, 2012 [citado 2013 junio 22]. Disponible en: http://www.ine.es/jaxi/menu.do?type=pcaxis\&path $=\% 2 \mathrm{Ft} 20 \% 2 \mathrm{Fe} 245 \&$ file $=$ inebase $\& \mathrm{~L}=0$

26. Instituto Nacional de Estadística y Geografía. Censo de población y vivienda 2010 [base de datos en Internet]. Explotación estadística de datos. Aguascalientes: INEGI, 2012 [citado 2013 junio 22]. Disponible en: http://www.inegi.org.mx/sistemas/olap/proyectos/bd/consulta.asp?p=17118\&c=27769\&s $=$ est\#

27. Kypri K, McAnally HM. Randomized controlled trial of a web-based primary care intervention for multiple health risk behaviors. Prev Med. 2005; 41:761766.

28. Carlson JA, Sallis JF, Ramirez ER, Patrick K, Norman GJ. Physical activity and dietary behavior change in Internet-based weight loss interventions: comparing two multiple-behavior change indices. Prev Med. 2012; 54:50-54.

29. Webb TL, Joseph J, Yardley L, Michie S. Using the internet to promote health behavior change: a systematic review and meta-analysis of the impact of theoretical basis, use of behavior change techniques, and mode of delivery on efficacy. J Med Internet Res. 2010; 12:e4.

30. Anderson-Bill ES, Winett RA, Wojcik JR. Social cognitive determinants of nutrition and physical activity among web-health users enrolling in an online intervention: the influence of social support, self-efficacy, outcome expectations, and self-regulation. J Med Internet Res. 2011;13(1):e28.

31. Aspy CB, Vesely SK, Oman RF, Tolma E, Rodine S, Marshall L, et al. School-related assets and youth risk behaviors: alcohol consumption and sexual activity. J Sch Health. 2012;82(1):3-10.

32. Edwards JU, Mauch L, Winkelman MR. Relationship of nutrition and physical activity behaviors and fitness measures to academic performance for sixth graders in a midwest city school district. J Sch Health. 2011;81(2):65-73.
33. Bramstedt KA. Recruiting healthy volunteers for research participation via Internet advertising. Clin Med Res. 2007;5:91-97.

34. Shubert TE, Altpeter M, Busby-Whitehead J. Using the RE-AIM framework to translate a research-based falls prevention intervention into a community-based program: lessons learned. J Safety Res. 2011; 42:509516.

35. Patel V, Nowostawski M, Thomson G, Wilson N, Medlin H. Developing a smartphone 'app' for public health research: the example of measuring observed smoking in vehicles. J Epidemiol Community Health. 2013; 67:446-452.

36. Rajani R, Berman DS, Rozanski A. Social networks--are they good for your health? The era of Facebook and Twitter. QJM. 2011; 104:819-820.

37. White A, Kavanagh D, Stallman H, Klein B, KayLambkin F, Proudfoot J, et al. Online alcohol interventions: a systematic review. J Med Internet Res. 2010; 12:e62.

38. Kostkova P, Farrell D, de Quincey E, Weinberg J, Lecky D, McNulty C, et al. eBug-teaching children hygiene principles using educational games. Stud Health Technol Inform. 2010; 160:600-604.

39. Google Analytics [Internet application]. Mountain View: Google Inc., 2013 [citado 20-05-2013]. Disponible en: http://www.google.com/analytics/.

40: Crutzen R, Roosjen JL, Poelman J. Using Google Analytics as a process evaluation method for Internetdelivered interventions: an example on sexual health. Health Promot Int. 2013;28:36-42.

41. Maes L, Cook TL, Ottovaere C, Matthijs C, Moreno LA, Kersting M, et al. Pilot evaluation of the HELENA (Healthy Lifestyle in Europe by Nutrition in Adolescence) Food-O-Meter, a computer-tailored nutrition advice for adolescents: a study in six European cities. Public Health Nutr. 2011;14(7):1292-302.

42. Beghin L, Huybrechts I, Vicente-Rodriguez G, DE Henauw S, Gottrand F, Gonzales-Gross M, et al. Mains characteristics and participation rate of European adolescents included in the HELENA study. Arch Public Health. 2012;70(1):14

43. Alff F, Markert J, Zschaler S, Gausche R, Kiess W, Blüher S. Reasons for (non)participating in a telephone-based intervention program for families with overweight children. PLoS One. 2012;7(4):e34580. 